\title{
QT dispersion and components of the QT interval in ischaemia and infarction
}

\author{
P D Higham, S S Furniss, R W F Campbell
}

\begin{abstract}
Objective-To evaluate changes in QT dispersion and components of the QT interval in patients admitted with unstable angina and acute myocardial infarction and to study the dynamics of these changes in patients with infarction. Methods-Prospective study recording electrocardiograms at $50 \mathrm{~mm} / \mathrm{s}$ in patients admitted with typical cardiac chest pain. Subsequent confirmation of acute myocardial infarction according to standard criteria. Single blind analysis for QT dispersion and QT components using a digitiser and simple computer program. Results are expressed as native QT dispersion, QTc dispersion, and the QT dispersion ratio defined as QT dispersion divided by cycle length and expressed as a percentage.
\end{abstract}

Results-QT dispersion, QTc dispersion, and QT dispersion ratio were all higher in patients with acute myocardial infarction than in those with unstable angina (mean (SD) 66 (18) ms, 75 (26) $\mathrm{ms}^{\frac{1}{2}}$, and $8 \cdot 1$ (2.4) \% compared with 38 (13) ms, 39 (13) $\mathrm{ms}^{\frac{1}{1}}$, and $4.5(1 \cdot 7) \%$ respectively). Dynamic changes in QTc dispersion were seen after acute infarction with significant differences in the $Q T$ components occurring between the different patient groups. Levels of QT dispersion (87 (15) ms), QTc dispersion (105 (17) $\left.\mathrm{ms}^{\frac{1}{2}}\right)$, and QT dispersion ratio $(11.7(0.8) \%)$ in the four patients with ventricular fibrillation were significantly higher. Use of QT dispersion ratio gave a narrower confidence interval.

Conclusion-QT dispersion is increased after myocardial infarction and levels are higher in patients with ventricular fibrillation. The changes in QT dispersion are dynamic and may reflect the changing pattern of underlying ventricular recovery of ventricular excitability, which is profoundly disturbed in the earliest phase of acute infarction. Expressing QT dispersion as a percentage of cycle length (QT dispersion ratio) rather than using standard rate correction may be superior in identifying patients who develop ventricular fibrillation.

(Br Heart f 1995;73:32-36)

Keywords: QT interval; infarction; ventricular fibrillation

Great effort has been expended on attempts to predict ventricular fibrillation in patients in the earliest phase of myocardial infarction. This arrhythmia remains the major source of mortality from myocardial infarction but with continuous electrocardiographic monitoring and the rapid availability of defibrillators, few patients with infarction die from ventricular fibrillation in hospital. Perhaps for this reason or because of the lack of success in the prediction of ventricular fibrillation, this problem has attracted relatively little recent attention.

Various features are significantly associated with primary ventricular fibrillation: time from onset of symptoms, ${ }^{1}$ size of infarction, ${ }^{2}$ potassium levels, ${ }^{3}$ etc, but none has clinically useful positive predictive accuracy. R-on-T ventricular extrasystoles have been investigated as predictors of ventricular fibrillation. ${ }^{4}$ They appeared an attractive prospect given their part in initiating primary ventricular fibrillation but their presence and frequency proved neither specific nor sensitive in predicting this arrhythmia. Whilst the practical clinical value of primary ventricular fibrillation prediction in acute myocardial infarction may be debatable, accurate prediction of the event would at least indicate a level of understanding of arrhythmogenesis that has, so far, been lacking.

Dispersion of repolarisation is an important electrophysiological feature which is considered fundamental for the initiation of ventricular fibrillation. ${ }^{56}$ Its predictive potential has not been examined because, until recently, measures of dispersion of repolarisation were not available in clinical practice. With recent evidence that differences in interlead QT intervals on standard twelve lead electrocardiograms (ECGs) reflect not technical artefact but regional variations in recovery of ventricular excitability, ${ }^{7}$ a method for examining repolarisation features as predictors of ventricular fibrillation has become available. This study examines QT dispersion in patients with acute myocardial infarction and with unstable angina. It explores the value of native versus rate corrected $\mathrm{QT}$ values and compares these with the use of a simple formula which expresses QT dispersion as a percentage of cycle length, thus obviating the need for rate correction. It further examines the dynamic behaviour of QT dispersion in acute myocardial infarction and describes QT changes which precede ventricular fibrillation.

Patients and methods

Standard twelve lead ECGs recorded at 50 $\mathrm{mm} / \mathrm{s}$ were obtained prospectively in patients admitted to the coronary care unit who were within $6 \mathrm{~h}$ of the onset of typical cardiac chest 
pain. Patients with manifest arrhythmia, bundle branch block and those taking antiarrhythmic agents or other medication likely to affect the QT interval were excluded. QT analysis was performed using a Calcomp 9000 digitizing tablet and a simple personal computer based computer program, as previously described. ${ }^{8}$ The end of the $T$ wave was defined visually as the point where the $T$ wave returned to the TP baseline. The apex of the $T$ wave was defined as the peak of the $T$ wave and in the case of biphasic $T$ waves the peak of the largest $\mathrm{T}$ wave component. Where the $T$ wave was interrupted by a $U$ wave before a return to baseline, the QT interval was measured to the nadir between the $T$ and $U$ waves. These definitions are in accordance with previously published work. ${ }^{8}$ As far as possible three complexes were analysed for each lead and all 12 leads were analysed. Leads where the $T$ wave end could not be discerned were excluded from the analysis. ECGs with less than seven leads available for
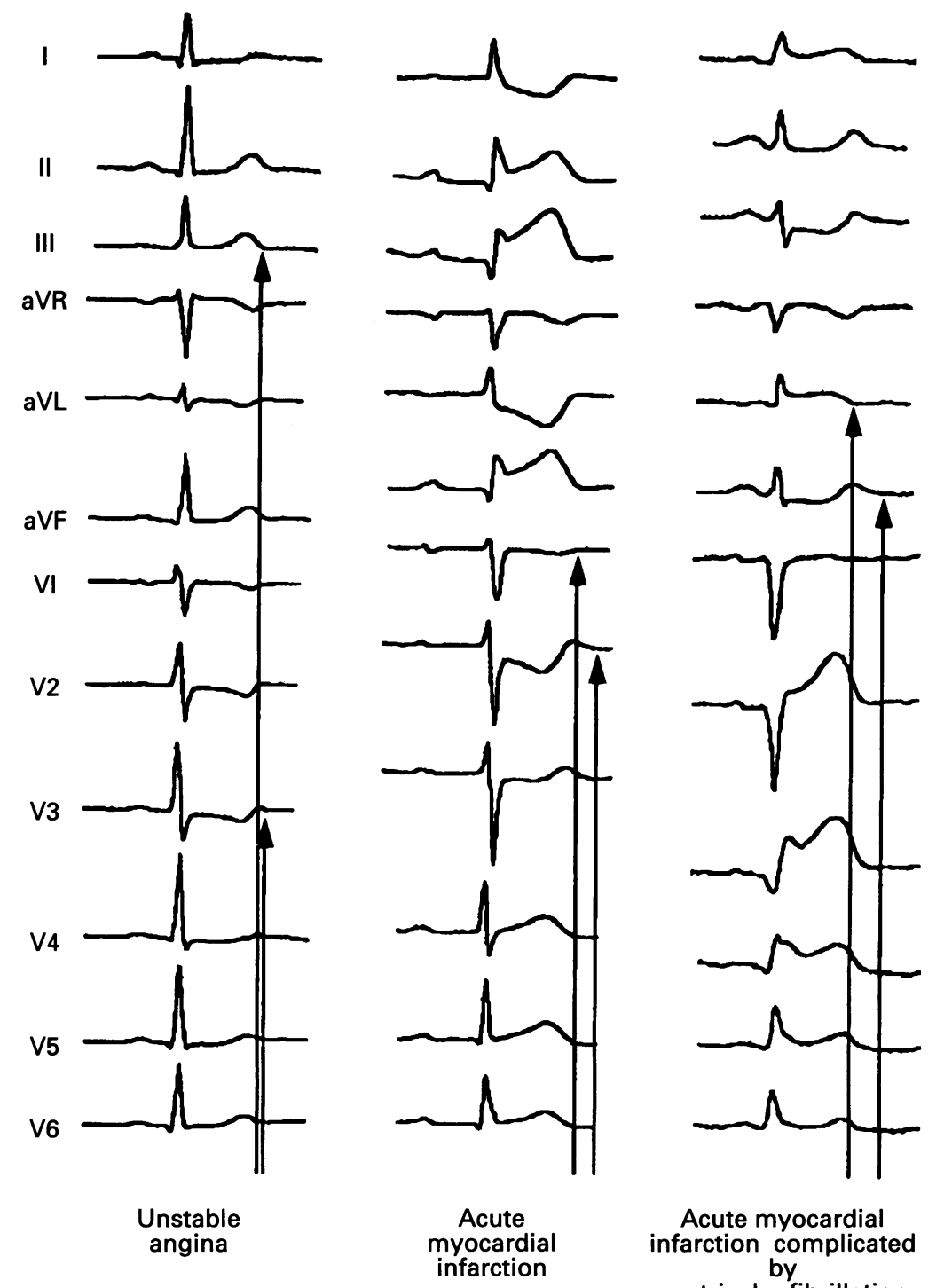

analysis were excluded $(n=2)$. The second ECG (usually performed the following day) in patients with confirmed myocardial infarction was also recorded at $50 \mathrm{~mm} / \mathrm{s}$ to document changes in QT dispersion with time.

Acute myocardial infarction was defined according to at least two of the three World Health Organisation criteria:

(a) typical cardiac chest pain for $>30 \mathrm{~min}$;

(b) serial ECG changes;

(c) peak creatine kinase >twice the upper limit of normal.

Unstable angina was diagnosed on the basis of typical acute cardiac chest pain with ST segment changes but without either $Q$ waves or a rise in cardiac enzymes; or typical acute chest pain with subsequent predischarge positive exercise test; or significant coronary artery disease (defined as one or more stenoses $>70 \%$ ) as shown by coronary angiography.

Results for QT dispersion are given as native values (QT dispersion), rate corrected using Bazett's formula (QTc dispersion), and the QT dispersion ratio which we have defined as QT dispersion divided by the cycle length in milliseconds and expressed as a percentage. Results are given as mean (SD) with statistical analysis performed using Student's $t$ test and the paired $t$ test for comparisons within patients.

\section{Results}

A total of 55 patients were enrolled into the study. Twenty five patients had unstable angina and 30 acute myocardial infarction, four of whom had an episode of primary ventricular fibrillation; all were successfully resuscitated.

The mean (SD) (range) heart rate expressed as cycle length in patients with unstable angina was 887 (167) (640-1200) $\mathrm{ms}$ and in those with acute infarction the values were similar with a mean cycle length of 824 (169) (610-1300) ms. The number of electrocardiography leads analysed in each group was also similar, averaging $10 \cdot 4$ for patients with unstable angina and 10.3 for those with infarction.

OT DISPERSION IN ISCHAEMIA AND INFARCTION The mean (SD) admission QT dispersion in patients with unstable angina was significantly lower (38 (13) $\mathrm{ms}$ ) than in those with myocardial infarction (69 (19) $\mathrm{ms}) \quad(P<0.001)$. The results were similar using rate corrected values with mean (SD) QTc dispersion values of 39 (14) $\mathrm{ms}^{\frac{1}{2}}$ for patients with unstable angina and 79 (27) $\mathrm{ms}^{\frac{1}{2}}$ for those with infarction $(P<0.001)$. The $Q T$ dispersion ratio was considerably higher in patients with acute infarction $(8.6(2.6) \%)$ than in those with unstable angina $(4.5(1.7) \%)(P<0.001)$.

There was no significant difference in QT dispersion between anterior $(n=14)$ and inferior $(n=16)$ infarcts with QT dispersion values of $70(21) \mathrm{ms}$ versus 68 (18) ms; mean QTc dispersion values of $84 \mathrm{~ms}^{\frac{1}{2}}$ and $79 \mathrm{~ms}^{\frac{1}{3}}$; and QT dispersion ratio values of $8.3(3.5) \%$ versus $8 \cdot 8(2 \cdot 8) \%$ respectively.

Figure 1 Electrocardiograms aligned to the onset of the QRS complex from patients with unstable angina, myocardial infarction and ventricular fibrillation illustrating dispersion of the $Q T$ intervals.
Acute myocardial Aarction complica by complicated 

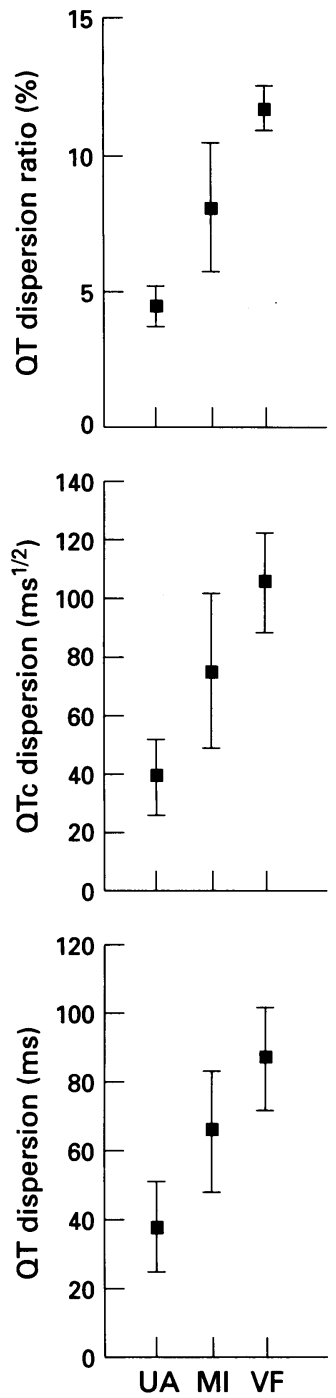

Figure 2 Admission $Q T$ dispersion, OTc dispersion dispersion, $Q T c$ dispersion,
and $Q T$ dispersion ratio in patients with unstable angina (UA), acute myocardial infarction (MI), and acute myocardial infarction complicated by ventricular fibrillation (VF). Bars are SD.
QT DISPERSION AND VENTRICULAR FIBRILLATION

Figure 1 shows representative electrocardiograms from patients with unstable angina, myocardial infarction, and myocardial infarction complicated by ventricular fibrillation annotating the QT dispersion for each. Figure 2 shows the results for QT dispersion, QTc dispersion, and the QT dispersion ratio illustrating the means (SD) for each parameter for patients with unstable angina and acute myocardial infarction with and without ventricular fibrillation. Native QT dispersion values were greater in the four patients who developed ventricular fibrillation (mean (SD) 87.5 (15) $\mathrm{ms}$ ) than in those without ventricular fibrillation complicating their acute myocardial infarction (mean (SD) $66(18) \mathrm{ms}(\mathrm{P}<$ $0.04)$. The results were again similar using rate corrected values, with QTc dispersion values of 75 (26) $\mathrm{ms}^{\frac{1}{2}}$ for patients without ventricular fibrillation and 105 (17) $\mathrm{ms}^{\frac{1}{2}}$ for those with the arrhythmia $(P<0.04)$. The mean QT dispersion ratio in patients with myocardial infarction who developed ventricular fibrillation was $11.7(0.8) \%$ versus $8.1(2.4) \%$ in those patients with infarction who did not have ventricular fibrillation. Figure 3 illustrates the differences between the means and confidence intervals for each parameter for comparison between patients with and without ventricular fibrillation who had a myocardial infarction. Four of seven patients with a QT dispersion ratio of $11 \%$ or greater had ventricular fibrillation with no patient having ventricular fibrillation below this value.

CHANGES IN QTC DISPERSION WITH TIME AFTER ACUTE MYOCARDIAL INFARCTION

A second ECG was recorded within $24 \mathrm{~h}$ in 27 of the 30 patients with acute infarction. Data for the three other patients were missing because of non-collection for two patients and the death of one from cardiogenic shock $3 \mathrm{~h}$ after admission. ECGs from four of the 27 patients were not included in the analysis: the ECG was taken during a further episode of chest pain in two patients (QTc dispersion increased in both by $30 \mathrm{~ms}^{\frac{1}{2}}$ ) and the other two patients developed right bundle branch block (QT dispersion increased only by 10 $\left.\mathrm{ms}^{\frac{1}{2}}\right)$. The ECGs from the remaining 23 patients were used to determine time dependent changes in QTc dispersion after acute

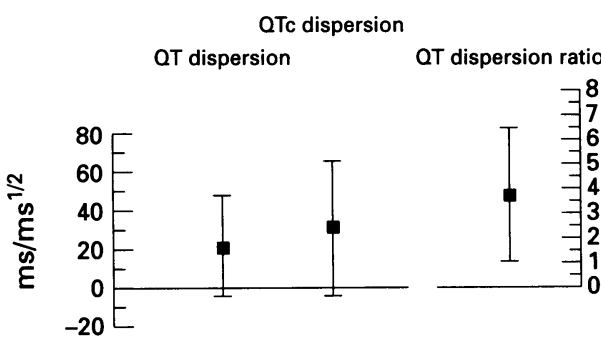

Figure 3 Comparison of the differences between means and confidence intervals for $Q T$ dispersion, QTc dispersion, and $Q T$ dispersion ratio in patients with acute myocardial infarction with and without ventricular fibrillation. myocardial infarction. The ECG pairs were a mean of 5 and $19 \mathrm{~h}$ after the onset of symptoms. There was a small but significant decrease in heart rate between the two recordings (mean cycle length change $=+57$ (114) ms, $P<0.02)$. QTc dispersion fell in 18 patients $(67 \%)$, remained unchanged in three and rose by $10 \mathrm{~ms}^{\frac{1}{2}}$ in three (fig 4). The mean change in the 24 patients was a significant reduction of $24 \mathrm{~ms}^{\frac{1}{2}}(\mathrm{P}<0.01)$.

\section{COMPONENTS OF QTC DISPERSION}

Figure 5 shows the mean QTc minimum and mean QTc maximum together with the rate corrected dispersion values for both the apex and end of the $T$ wave in patients with unstable angina, myocardial infarction, and ventricular fibrillation.

QTc end dispersion was increased in patients with acute myocardial infarction and was significantly higher in patients with ventricular fibrillation. There was no significant difference in QT end minimum in patients with infarction compared with that in those with ventricular fibrillation or unstable angina. Thus, QTc end dispersion was largely generated by prolongation of QTc end maximum.

Similar changes in QTc apex dispersion were found-that is, QTc apex dispersion was significantly greater in patients with infarction than in those with unstable angina, but QTc apex did not distinguish those patients who would develop ventricular fibrillation. By contrast with analysis of the minimum component of QTc end dispersion, QTc apex minimum was shorter in patients with infarction and largely responsible for the increased QTc apex dispersion in that group.

\section{Discussion}

This paper represents a detailed examination of QT dispersion parameters in patients admitted to the coronary care unit in an attempt to define whether refinement of measurement of the traditional single QT interval will identify patients at risk of ventricular fibrillation. In considering QT rate correction we have elected to use Bazett's formula and to

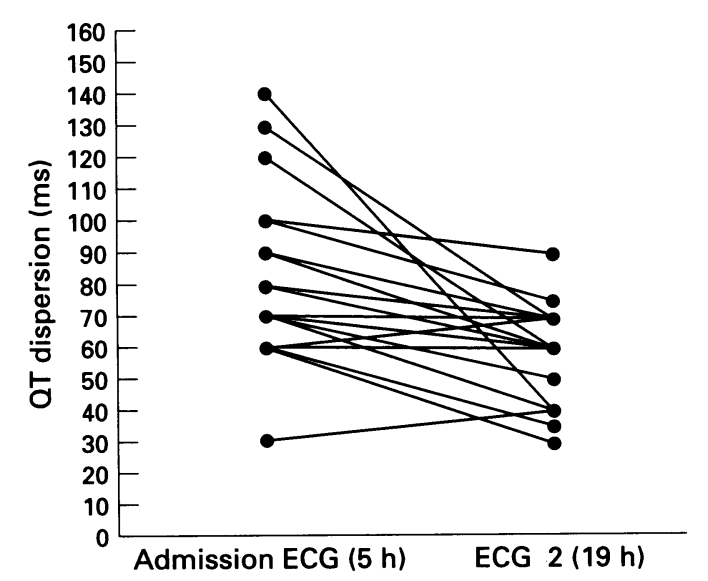

Figure 4 Changes in $Q T c$ dispersion with time after acute myocardial infarction. ECG, electrocardiogram. 
Figure 5 Changes in $Q T c$ components in patients with ischaemia and infarction. $* P<0.05$ $\star \star P<0.01 ; * \star \star P P<0.001$ 圆, QTc end maximum; $\square$, QTc end minimum; Q QTc apex maximum; -, QTc apex minimum; , QTC apex dispersion $\mathbb{\mathbb { N } , Q T c}$ end dispersion. Bars are $S E$.

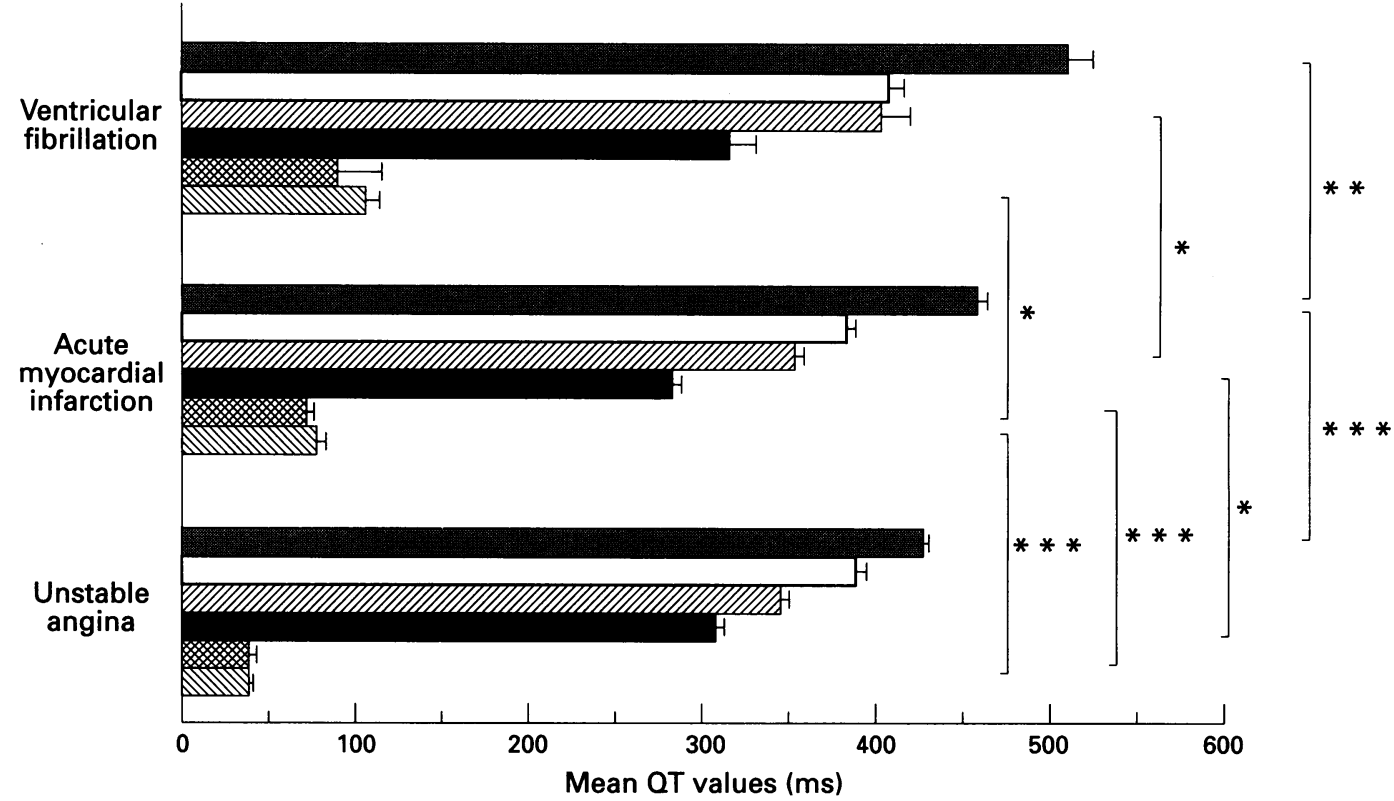

compare this with the native values and with a newly defined parameter, namely, the QT dispersion ration. QT rate correction suffers from many problems, not least the fact that nearly all the methods have been derived from observations made in healthy subjects and thus are probably inappropriate in the context of acute infarction.

This paper provides direct evidence of important QT component changes with infarction as compared with those of ischaemia, suggesting that the approach is invalid. The use of the QT dispersion ratio may offer a better approach. It avoids the need for rate correction and offers a direct measurement of the time per cycle length for which the underlying recovery of ventricular excitability is disturbed.

This is also the first detailed examination of QTc components (QTc end and QTc apex, QTc maximum and QTc minimum) and QT dynamics in a substantial ischaemic population. The complexity of the changes associated with unstable angina, infarction, and infarction complicated by ventricular fibrillation may help explain the often contradictory descriptions of the QTc interval as a risk factor after acute myocardial infarction. In some studies, ${ }^{910}$ prolongation of the QTc interval was associated with ventricular fibrillation. Other work, ${ }^{11}$ however, however, has been contradictory. The lack of standard criteria for measuring QT intervals, the differing measurement protocols used, and the failure to take account of the differences between different leads may explain these inconsistencies.

This study did not examine a normal population group, but in other work, ${ }^{812}$ a normal QTc end dispersion of about $50 \mathrm{~ms}^{\frac{1}{2}}$ has been described. Patients with unstable angina show admission values close to the normal range (mean $39 \mathrm{~ms}^{\frac{1}{2}}$ ). Patients with acute infarction have higher admission QTc end dispersion (mean $75 \mathrm{~ms}^{\frac{1}{2}}$ ) with mean QTc end dispersion values of $105 \mathrm{~ms}^{\frac{1}{2}}$ in patients with ventricular fibrillation. Prolongation of QTc end maximum rather than shortening of QTc end minimum was responsible for the increase in QTc end dispersion in patients with myocardial infarction. In those who developed ventricular fibrillation greater prolongation of QTc end maximum generated the increased dispersion associated with this arrhythmia. QTc end measures the completion of repolarisation from the body surface. It is influenced by both myocardium that repolarises late or even fails to repolarise, thus creating boundary currents, and significant populations of cells with after depolarisations. All of these factors may contribute to the development of fibrillation by disrupting the normally uniform recovery of the ventricle.

It is difficult to explain the nuances of QTc apex changes. QTc apex minimum shortens after acute myocardial infarction compared with that in patients with unstable angina. This effect is still present after excluding the two patients in either category who had biphasic T waves. Little is understood of what QTc apex represents but in the homogeneous myocardium with a symmetrical $\mathrm{T}$ wave, the $T$ apex might be expected to represent the point at which $50 \%$ of myocardium has repolarised. Thus QTc apex measurements will in part be determined by shortening of action potentials due to ischaemia and infarction. This may explain the insensitivity of QTc apex dispersion measurements to differentiate patients with ventricular fibrillation.

QTc end dispersion falls quickly in most patients after infarction. Twenty six of our patients were treated with streptokinase and some of the larger falls may have been the result of reperfusion which reduces QTc dispersion. ${ }^{13}$ With the widespread use of thrombolytic agents it will be difficult to establish the natural history of changes in QT dispersion after infarction but a study of retrospective data should be possible. Drugs may also influence QT dispersion. Patients taking medication known to affect the QT interval or antiarrhythmic drugs were excluded from this 
study but nothing is known of the effect of $\beta$ blocking agents or other commonly used drugs on QT dispersion.

In previous work Day et $a^{14}$ used adjusted QTc dispersion to correct for the different number of leads analysed. Adjusted dispersion is derived by dividing the QTc dispersion value by the square root of the number of leads analysed. We have not used this correction for two reasons. First, the number of leads analysed was similar in all the groups (mean $=10$ ) and at least seven leads had to be suitable for analysis, thus reducing the need to perform such an adjustment. Second, the mathematical basis of adjusted QTc dispersion assumes that all leads are equally likely to contribute to QTc minimum and QTc maximum; an assetion that may be flawed.

This study was not designed to be the definitive examination of QT dispersion as a risk factor for ventricular fibrillation but rather to provide information that would permit design of such a study. Only four of the consecutively included ischaemic patients developed ventricular fibrillation but even in this very small group, significant QT measurement deviations were seen. The confidence limits are large for QT dispersion and QTc dispersion and extend beyond zero but are promising with the use of the QT dispersion ratio. A larger study will be necessary to determine accurately its predictive power. The dynamic nature of changes in QTc end dispersion imply that the admission ECG alone may not provide sufficient information. Real time monitoring of the interlead differences between QT intervals may have greater value as rapid changes in dispersion of ventricular recovery are probably more important that a single static value. Such a monitoring system should accurately track changes in QT end maximum and QT end minimum. Changes in the lead position of QT maximum or QT minimum with further ischaemia and/or extension of infarction may provide added difficulties and thus determination of the optimum number and position of leads to monitor will be important. Combined monitoring of both QT end dispersion and R-on-T ventricular extrasystolic activity may be an even more sensitive method for prediction of ventricular fibrillation with one method (QT dispersion) focusing on the arrhythmia substrate and the other (R-on-T extrasystokes) focusing on the arrhythmia trigger. Automated systems are necessary and improved algorithms for QT end detection required, however, to perform such monitoring.

PDH was a recipient of a British Heart Foundation Junior Research Fellowship. The Academic Department of Cardiology, Freeman Hospital, Newcastle upon Tyne is supported by the British Heart Foundation.

1 Pantridge JF, Web SW, Adgey AAJ. Arrhythmias in the first 12 hours of acute myocardial infarction. Prog first 12 hours of acute myocar

2 Grande P, Kiilreich S. Relationship between serum CK-M estimated acute myocardial infarct size and clinical complications. Acta Med Scand 1984;215:355-62.

3 Norderehaug JE, Von der Lippe G. Hypokalaemia and ventricular fibrillation in acute myocardial infarction. $B$ Heart $\mathcal{f} 1983 ; 50: 525-9$.

4 El-Sherif N, Myerburg RJ, Scherlag BJ, Befeler B, Avanda JM, Castellanos A, Lazzara R. Electrocardiographic antecedents of primary ventricular fibrillation. Value of the R-on- $\mathrm{T}$ phenomenon in myocardial infarction. $B r$ Heart f 1976;38:415-22.

5 Pogwizd SM, Corr PB. Mechanisms underlying the development of ventricular fibrillation during early myocardial ischaemia. Circ Res 1990;66:672-95.

6 Janse MJ, Wit AL. Electrophysiological mechanisms of ventricular arrhythmias resulting from myocardial ischaemia and infarction. Physiol Rev 1989;69: ischaemia

7 Higham PD, Hilton CJ, Aitcheson DA, Furniss SS, Bourke JP, Campbell RWF. QT dispersion does reflect Bourke JP, Campbell RWF. QT dispersion does reflect Circulation 1992;86(suppl):I392.

8 Cowan JC, Yusoff K, Moore M, et al. Importance of lead selection in QT interval measurement. Am $f$ Cardiol 1988;61:83-7.

9 Taylor GJ, Crampton RS, Gibson RS, et al. Prolonged QT interval at onset of acute myocardial infarction in predicting early phase ventricular tachycardia. Am Heart $\mathcal{F}$ 1981;102:16-24.

10 Ahnve S, Lundman T, Shoaleh-var M. The relationship between QT interval and ventricular arrhythmias in acute myocardial infarction. Acta Med Scand 1978;204 17-9.

11 Giglioli C, Margheri M, Bartoletti A, et al. Valore dell'intervallo QT como segno premonitore di gravi aritmie ventricolari nellla fase precoce dell'infarto miocardico. G Ital Cardiol 1988;18:290-5.

12 Sylvein JC, Horacek BM, Spencer CA, Klassen GA, Montague TJ. QT interval variability on the body surMontague TJ. QT interval variability

13 Higham PD, Reid DS, Campbell RWF, Furniss SS. Reperfusion, acute myocardial infarction and QT Reperfusion, acute myocardial infarction and QT
dispersion [abstract]. Eur Heart $\mathcal{1}$ 1992;13(suppl):448.

dispersion [abstract]. Eur Heart f 1992;13(suppl):448.
14 Day CP, McComb JM, Matthews J, Campbell RWF Reduction in QT dispersion by sotalol following myocardial infarction. Eur Heart $\mathcal{f}$ 1991;12:423-7. 